\title{
A Special Case Report of Aggressive Hemorrhage in Posterior Vaginal Fornix DIE
}

\author{
Junling Liu, Dan Tang, Ruxia Shi, Yunfen Jiang, Zhongfang Zhang and Jiming Chen* \\ Department of Obstetrics and Gynecology, the Affiliated Changzhou NO. 2 People's Hospital of Nanjing Medical University, Changzhou 213000, China
}

*Corresponding author: Jiming Chen, Department of Obstetrics and Gynecology, the Affiliated Changzhou No. 2 People's Hospital of Nanjing Medical University, Changzhou 213000, Jiangsu Province, China.
Received Date: November 13, 2018

Published Date: November 27, 2018

\section{Introduction}

Deep infiltrating endometriosis (DIE) is defined by the presence of endometrial implants, fibrosis, and muscular hyperplasia below the peritoneum, invading the tissue to a depth of more than $5 \mathrm{~mm}$ [1]. Deep endometriosis involves, in descending order of frequency, the uterosacral ligaments (USL), the rectosigmoid colon, the vagina, and the bladder [2]. Pain and infertility are the main manifestations of DIE, including dysmenorrhea pain, chronic pelvic pain, dyspareunia, defecation pain, dysuria and back pain. DIE lesions are deep infiltration, severe pain symptoms, poor conservative treatment, difficult surgery, pelvic adhesions, and high risk of surgical accessory injury. It has been a difficult point in clinical treatment of endometriosis and gynecological laparoscopic surgery, there is no unified clinical type and treatment specifications [3-4]. This paper reported a special case of recurrent vaginal hemorrhage caused by posterior vaginal fornix DIE.

\section{Case Report}

Female, 31-year-old, married, 1-0-1-1, hospitalized for "vaginal bleeding for 3 hours". Regular menstruation, 7/30d, moderate menstruation, no dysmenorrhea, normal leucorrhea, LMP:2017-101. At 9:00am on December 2, 2017, without obvious inducement, there was massive vaginal hemorrhage, accompanied by blood clots. B ultrasound examination was done in Affiliated Changzhou No.2 People's Hospital of Nanjing Medical University finding inhomogeneous posterior cervical masses. It may be trophoblastic disease. Cervical pregnancy remains to be checked. Gynecological examination: vulva, married type; vagina: unimpeded, no excreta; uterine neck: smooth, no lift pain, a rupture, about $0.2 \mathrm{~cm}$ in diameter, was seen in the posterior cervical fornix, active hemorrhage; uterus: meso-position, full, regular, no pressing pain; adnexa: there was no obvious abnormality in bilateral appendix area.

The patient was admitted to hospital for "cervical occupancy: cervical pregnancy?" We firstly used cotton ball to stop bleeding, and then took anti-infective therapy and auxiliary examinations. A biopsy of the posterior vaginal fornix rupture showed chronic inflammation with hemorrhage. Blood HCG: 2.39mIU/ml, excluding the possibility of cervical pregnancy. Cervical TCT showed atypical squamous cells that could not be defined. Cervical HPV showed that the HPV12 was positive and the others were negative, and the possibility of cervical cancer could not be excluded. Further cervical biopsy is needed.

The possibility of cervical tuberculosis was excluded according to no previous tuberculosis history, physical signs, and auxiliary examinations after admission. After admission, uterine arteriography was done thereby eliminating the possibility of vascular malformation. The patient finally underwent the laparoscopic surgery including posterior vaginal fornix lesion resection, pelvic adhesion separation, intestinal adhesion separation, cervical biopsy, and cervical tracheal scratch on December 14, 2017. The surgery was successful. Intraoperative rapid pathology indicated that there was chronic active inflammation with no malignant lesions in (cervical) mucosa. Postoperative routine pathology showed (cervical) mucosa chronic active inflammation and amount of broken tissues: endometriosis. Pathological findings of cervical biopsy: $(3,6,9,12$ points $)$ chronic active inflammation, focal condyloma of squamous epithelium. (Cervical canal scratches) A small amount of broken cervical tissues and mucus showed chronic inflammation. It was finally diagnosed as deep endometriosis of posterior vaginal fornix.

\section{Discussion}

Endometriosis is a condition in which the tissue of the endometrium (gland and stroma) appears outside the uterus. The ectopic endometrium can invade any part of the whole body, but the vast majority is located in pelvic viscera and wall peritoneum, the ovary and uterosacral ligaments are the most common, followed 
by uterus, visceral peritoneum, rectovaginal diaphragm, so it is called pelvic endometriosis [5]. Endometriosis shows a benign morphological appearance, but it is similar to malignant tumor in clinical behaviors, such as implantation, invasion and distant metastasis. Clinical manifestations mainly include lower abdominal pain and dysmenorrhea, infertility, sexual discomfort and menstrual abnormality. When any part outside the pelvic cavity is implanted with ectopic endometrial, periodic pain, hemorrhage and mass can occur locally, and corresponding symptoms appear. DIE is a group of endometriosis lesions infiltrating below the peritoneum with a depth of $5 \mathrm{~mm}$ or more [1], which can be located anywhere in the pelvic cavity. Common DIE lesions include pelvic posterior endometriosis, cystopathia and ureteral endometriosis [6]. This kind of deep endometriosis with the main clinical manifestation of posterior cervical fornix rupture hemorrhage is also a rare case in clinical practice, which requires us to consider comprehensively. After the differential diagnosis of diseases with similar symptoms, the possibility of DIE should be considered to avoid misdiagnosis and missed diagnosis.

\section{Acknowledgement}

None.

\section{Conflict of Interest}

No Conflict of Interest.

\section{References}

1. Bazot M, Lafont C, Rouzier R, Roseau G, Thomassin-Naggara I, et al. (2009) Diagnostic accuracy of physical examination, transvaginal sonography, rectal endoscopic sonography, and magnetic resonance imaging to diagnose deep infiltrating endometriosis. Fertil Steril 92(6): $1825-1833$

2. Dai Y, Leng JH, Lang JH, Li XY, Zhang JJ (2012) Anatomical distribution of pelvic deep infiltrating endometriosis and its relationship with pain symptoms. Chin Med 125(2): 209-213.

3. Berkley KJ, Rapkin AJ, Papka RE (2005) The pains of endometriosis. Science 308(5728): 1587-1589.

4. Valle RF, Sciarra JJ (2003) Endometriosis: treatment strategies. Ann N Y Acad Sci 997: 229-239.

5. Dai Y, Leng JH, Lang JH, Liu ZF, Li XY, et al. (2010) Clinico-pathologic characteristics of posterior deeply infiltrating endometriosis lesions, pain symptoms and its treatment using laparoscopic surgery. Zhonghua Fu Chan Ke Za Zhi 45(2): 93-98.

6. Zhang J, Leng J, Dai Y, Lang J (2014) Significance of symptom and physical sign to diagnosis of deeply infiltrating endometriosis. Zhonghua Fu Chan Ke Za Zhi 49(8): 599-603. 